\title{
Red Sox Versus Yankees, Sports Team Rivalry, Sports Symbols, and Distance Performance: An Abstract
}

\author{
Sukki Yoon, Austin Beltis, John Logan, Kacy Kim, and Gayatri Subramanian
}

\begin{abstract}
Building on the literature on psychological distance and visual illusion, the authors examine whether visual exposure to sports team symbols affects distance performance, depending on whether the symbols represent favored or rival teams. Boston Red Sox fans are used to test the hypothesis in the context of the famous Red Sox-Yankees rivalry. Individuals who are primed with the symbol of their favored (rival) sports team are predicted to hit/throw a ball short (long) of the target. In Study 1, Red Sox fans putt a golf ball imprinted with a Red Sox (Yankees) logo and are found to putt short (long) of the goal. In Study 2, Red Sox fans photograph a bobble head wearing a Red Sox (Yankee) jersey and then are found to throw a basketball nearer to (farther from) themselves.
\end{abstract}

References Available Upon Request

S. Yoon $(\bowtie) \bullet$ A. Beltis $\bullet$ J. Logan $\bullet$ G. Subramanian

Bryant University, Smithfield, RI, USA

e-mail: syoon@bryant.edu; austinbeltis@gmail.com; johndukelogan@gmail.com;

gsubra4@gmail.com

K. Kim

Elon University, Elon, NC, USA

e-mail: kkim3@elon.edu 